\title{
Effect of Co-Doping on Magnetic Properties of Bismuth Ferrite
}

\author{
I. Makoed ${ }^{a, *}$, V. Prigodich ${ }^{a}$, K. Yanushkevich ${ }^{b}$, A. Zhivulko ${ }^{b}$, V. Zhivulko ${ }^{b}$, \\ A. Galias $^{b}$, O. Demidenko ${ }^{b}$ And D. KRIVChenya ${ }^{b}$ \\ ${ }^{a}$ Brest State University named after A.S. Pushkin, Boulevard of Cosmonauts, 21, Brest, Republic of Belarus \\ ${ }^{b}$ Scientific-Practical Materials Research Centre NAS of Belarus, Laboratory of Physics of Magnetic Materials, \\ 220072, Minsk, P. Brovki str., 19, Belarus
}

\begin{abstract}
The effect of co-doping on structure and magnetic properties of the $\mathrm{BiFeO}_{3}$-based multiferroics with a partial isovalent substitution of bismuth for $\mathrm{La}^{3+}, \mathrm{Gd}^{3+}, \mathrm{Dy}^{3+}$, and $\mathrm{Er}^{3+}$ ions have been experimentally investigated by $\mathrm{X}$-ray diffraction and magnetic methods. The ceramic $\mathrm{R} 1_{x} \mathrm{R} 2_{0.2-x} \mathrm{Bi}_{0.8} \mathrm{FeO}_{3}$ type $(x=0,0.05,0.10,0.15,0.20$; $\mathrm{R} 1, \mathrm{R} 2=\mathrm{La}, \mathrm{Gd}, \mathrm{Dy}, \mathrm{Er})$ samples have been prepared by a solid-state reaction method under cold pressing at high pressure $P=4 \mathrm{GPa}$. Temperature dependences of magnetization for the co-doped $\mathrm{BiFeO}_{3}$ demonstrate magnetic "weak ferromagnetic-antiferromagnetic" phase transitions in a high temperature range $T=550-650 \mathrm{~K}$. The presence of a weak ferromagnetism in all compositions is confirmed by open loops of magnetic field dependences. It has been found out that the magnetic characteristics strongly depend on the degree of substitution, temperature, and magnitude of magnetic field.
\end{abstract}

DOI: 10.12693/APhysPolA.137.985

PACS/topics: multiferroics, Néel temperature, phase transition

\section{Introduction}

Multiferroics are the most promising materials, in which two or more types of ferroic ordering phenomena such as ferromagnetism, ferroelectricity, and ferroelasticity coexist [1]. The most well-known multiferroics are compounds obtained by partial substitution in $\mathrm{BiFeO}_{3}$ (BFO) of bismuth cations by isovalent rare-earth cations. In this case, the growth of the resulting magnetic moment and the enhancement of the magnetoelectric interaction is observed. It is interesting to study the mutual influence of various cations of rare-earth elements on the intensity of magnetic interactions and the type of magnetic ordering that forms in $\mathrm{BFO}$ with partial isovalent substitution of bismuth cations. For small $(x<0.10)$ degrees of substitution of $\mathrm{Bi}^{3+}$ cations with rare-earth $(\mathrm{R})$ cations, $\mathrm{RBFO}$ samples crystallize in the rhombohedral $R 3 c$ lattice, isostructural to $\mathrm{BFO}$ lattice [2]. A relatively small number of works are known in which data on the structure and physical properties of materials are obtained with $\mathrm{BFO}$ co-doped by cations La-Gd [3], La-Sm [4], La-Ho [5], La-Dy [6], La-Tb [7], and $\mathrm{La}-\mathrm{Ce}[8]$. The aim of this work is to study the crystal structure features and magnetic property correlations in multiferroics of $\mathrm{R} 1_{x} \mathrm{R}_{0.2-x} \mathrm{Bi}_{0.8} \mathrm{FeO}_{3}$ type $(x=0$, $0.05,0.10,0.15,0.20 ; \mathrm{R} 1, \mathrm{R} 2=\mathrm{La}, \mathrm{Gd}$, Dy, Er) depending on the concentrations and types of substitutive rare-earth elements cations.

*corresponding author; e-mail: igmak2010@yandex.by

\section{Experimental part}

The polycrystalline $\mathrm{R} 1_{x} \mathrm{R} 2\left({ }_{0.2-x} \mathrm{Bi}_{0.8} \mathrm{FeO}_{3}(x=0\right.$, $0.05,0.10,0.15,0.20 ; \mathrm{R} 1, \mathrm{R} 2=\mathrm{La}, \mathrm{Gd}, \mathrm{Dy}, \mathrm{Er})$ samples were synthesized by a solid-state reaction route [9]. The analysis and refinement of X-ray data were performed by JANA2006 software using the Rietveld method [10]. Magnetic-field dependences of a specific magnetization were studied by SQUID magnetometer LOT-Quantum Design MPMS-XL and a vibration magnetometer OXFORD at temperatures of 5, 10, 100, and $300 \mathrm{~K}$ in magnetic fields up to $H=8 \mathrm{~T}$. The temperature dependences of the magnetization for the samples placed in vacuum quartz ampoules were measured by the Faraday method [11] within the temperature range of $T=300-1000 \mathrm{~K}$ in a magnetic field of $H=0.86 \mathrm{~T}$.

\section{Results and discussions}

The results of full-profile analysis of X-ray patterns of LGBFO and DEBFO samples according to the Rietveld method are given in Table I and Table II. Based on the data obtained, it can be concluded that co-doping of $\mathrm{BFO}$ with $\mathrm{Dy}^{3+}$ and $\mathrm{Er}^{3+}$ cations leads to stabilization of crystal lattices and to obtaining samples with a predominant Pnma phase. The amount of $\mathrm{Bi}_{2} \mathrm{Fe}_{4} \mathrm{O}_{9}$ impurity, which does not exhibit ferromagnetic, ferrimagnetic, and ferroelectric properties at temperatures higher than $250 \mathrm{~K}$ [12], is significantly lower during co-doping than what is observed in pure BFO. It is very difficult to obtain the pure phase in co-doped $\mathrm{BiFeO}_{3}$ due to kinetics of formation, which is attributed to a loss of the volatile 
TABLE I

The lattice parameters $(\AA)$ and unit cell volume $\left(\AA^{3}\right)$ for the $\operatorname{La}_{x} \operatorname{Gd}_{0.2-x} \mathrm{Bi}_{0.8} \mathrm{FeO}_{3}(x=0,0.05,0.10,0.15,0.20)$ samples obtained by the Rietveld refinement of X-ray patterns.

\begin{tabular}{|c|c|c|c|c|c|c|c|c|}
\hline \multirow{2}{*}{ Composition } & \multirow{2}{*}{$\begin{array}{l}\text { Space } \\
\text { group }\end{array}$} & \multicolumn{7}{|c|}{ Structural parameters and criteria of fit } \\
\hline & & $a[\AA]$ & $b[\AA]$ & $c[\AA]$ & $V\left[\AA^{3}\right]$ & GOF & $R_{\mathrm{p}}[\%]$ & $R_{\mathrm{wp}}[\%]$ \\
\hline $\mathrm{Gd}_{0.2} \mathrm{Bi}_{0.8} \mathrm{FeO}_{3}$ & Pnma & 5.6200 & 7.7992 & 5.4239 & 237.7 & 1.08 & 11.83 & 17.23 \\
\hline $\mathrm{La}_{0.05} \mathrm{Gd}_{0.15} \mathrm{Bi}_{0.8} \mathrm{FeO}_{3}$ & Pnma & 5.6300 & 7.8004 & 5.4462 & 239.3 & 1.16 & 13.25 & 19.50 \\
\hline $\mathrm{La}_{0.10} \mathrm{Gd}_{0.10} \mathrm{Bi}_{0.8} \mathrm{FeO}_{3}$ & Pnma & 5.5812 & 7.9021 & 5.4541 & 240.5 & 1.13 & 16.16 & 23.40 \\
\hline $\mathrm{La}_{0.15} \mathrm{Gd}_{0.05} \mathrm{Bi}_{0.8} \mathrm{FeO}_{3}$ & Pnma & 5.6058 & 7.8234 & 5.5755 & 244.5 & 0.99 & 10.70 & 16.76 \\
\hline $\mathrm{La}_{0.2} \mathrm{Bi}_{0.8} \mathrm{FeO}_{3}$ & $C 222$ & 5.7666 & 7.9306 & 5.5871 & 255.5 & 1.17 & 13.14 & 17.78 \\
\hline
\end{tabular}

TABLE II

The lattice parameters $(\AA)$ and unit cell volume $\left(\AA^{3}\right)$ for the $\operatorname{Dy}_{x} \operatorname{Er}_{0.2-x} \operatorname{Bi}_{0.8} \mathrm{FeO}_{3}(x=0,0.05,0.10,0.15,0.20)$ samples obtained by the Rietveld refinement of X-ray patterns.

\begin{tabular}{l|c|c|c|c|c|c|c|c}
\hline \hline \multirow{2}{*}{ Composition } & \multirow{2}{*}{$\begin{array}{c}\text { Space } \\
\text { group }\end{array}$} & \multicolumn{7}{|c}{ Structural parameters and criteria of fit } \\
\cline { 3 - 8 } & $a[\AA]$ & $b[\AA]$ & $c[\AA]$ & $V\left[\AA^{3}\right]$ & GOF & $R_{\mathrm{p}}[\%]$ & $R_{\mathrm{wp}}[\%]$ \\
\hline $\mathrm{Er}_{0.2} \mathrm{Bi}_{0.8} \mathrm{FeO}_{3}$ & Pnma & 5.7666 & 7.9306 & 5.5871 & 255.5 & 1.15 & 13.14 & 17.78 \\
$\mathrm{Dy}_{0.05} \mathrm{Er}_{0.15} \mathrm{Bi}_{0.8} \mathrm{FeO}_{3}$ & Pnma & 5.7715 & 7.9401 & 5.6126 & 257.2 & 0.99 & 12.05 & 16.12 \\
$\mathrm{Dy}_{0.10} \mathrm{Er}_{0.10} \mathrm{Bi}_{0.8} \mathrm{FeO}_{3}$ & Pnma & 5.7175 & 7.9154 & 5.5908 & 253.0 & 0.96 & 11.89 & 16.84 \\
$\mathrm{Dy}_{0.15} \mathrm{Er}_{0.05} \mathrm{Bi}_{0.8} \mathrm{FeO}_{3}$ & Pnma & 5.4186 & 7.9379 & 5.6026 & 241.0 & 0.96 & 11.67 & 15.39 \\
$\mathrm{Dy}_{0.2} \mathrm{Bi}_{0.8} \mathrm{FeO}_{3}$ & Pnma & 5.4196 & 7.8977 & 5.6216 & 240.6 & 1.09 & 12.64 & 16.96
\end{tabular}

bismuth during the calcination process. The content of $\mathrm{Bi}_{2} \mathrm{Fe}_{4} \mathrm{O}_{9}$ impurity in the $\mathrm{La}_{0.2} \mathrm{Bi}_{0.8} \mathrm{FeO}_{3}$ sample is maximum and amounts to $9.2 \%$. Traces of the complex oxide $\mathrm{Bi}_{25} \mathrm{FeO}_{39}$ were detected, the content of which does not exceed $3 \%$ in any of the studied samples. Although the obtained values of the lattice parameter, differ in absolute magnitude from known literature data, the concentration dependences of their values agree well with the results obtained in independent studies [3].

The introduction of $\mathrm{La}^{3+}$ cations with increase in substitution degree causes a gradual structural distortion, and for $x>0.15$, to a transformation from the orthorhombic to rhombic $C 222$ lattice. As can be seen in Table I, with an increase in the content of $\mathrm{Gd}^{3+}$ cations in the LGBFO system, the values of the volumes of unit cells decrease. This is due to the replacement of larger $\mathrm{La}^{3+}$ cations $(1.032 \AA)$ by smaller $\mathrm{Gd}^{3+}(0.938 \AA)$ ions [13]. The criteria of fit $\mathrm{La}_{0.2} \mathrm{Bi}_{0.8} \mathrm{FeO}_{3}$ sample for Pnma, $R 3 c$ space group were $\mathrm{GOF}=1.83, R_{\mathrm{p}}=18.17 \%$, $R_{\mathrm{wp}}=27.92 \%, \mathrm{GOF}=1.86, R_{\mathrm{p}}=19.04, R_{\mathrm{wp}}=28.45$, respectively. In compositions with $x<0.15$, the Pnma phase predominates. Accordingly, the decrease in the volume of unit cells is more pronounced in samples with high concentrations of $\mathrm{Gd}^{3+}$ ions. The results of the data analysis presented in Table II indicate that the magnitudes of the constant lattices of the DEBFO samples are systematically changed due to the $4 f$-compression effect, but the structural factors are slightly sensitive to small changes in the composition. As the degree of substitution increases, the diffraction maxima shift to higher angles. However, since the radii of the $\mathrm{Dy}^{3+}$ and $\mathrm{Er}^{3+}$ cations are close, the values of the parameters of the crystal lattices of the samples vary slightly depending on the substitution degree. This circumstance suggests that changes in the magnetic properties of DEBFO samples will be more determined by the magnetic characteristics of the substituting cations than by changing the parameters of their crystal lattices. The absolute magnitude of the intrinsic magnetic moment of the $\mathrm{Dy}^{3+}$ cation $\left(10.7 \mu_{\mathrm{B}}\right)$ is larger than that of $\operatorname{Er}^{3+}\left(9.5 \mu_{\mathrm{B}}\right)$ ones [14]. Therefore, it is logical to expect the observation of higher values of specific magnetizations with increasing degree of substitution at least at low temperatures. The magnetization curves of the samples exhibit hysteresis characteristic of ferromagnetic materials. Unlike pure $\mathrm{BiFeO}_{3}$, which shows a linear dependence of the specific magnetization on the magnitude of the magnetic field typical of antiferromagnets, the magnetic field dependences of cationsubstituted samples indicate the presence of a weak ferromagnetic moment. In the case of substitutions of $\mathrm{Bi}^{3+}$ ions, quantitative changes in the values of specific magnetizations and values of coercive fields recovered from particular magnetic hysteresis loops are observed, as can be seen in Fig. 1a-d.

Such behavior is revealed by the concentration dependences of the magnetic characteristics of the samples of the $\mathrm{Dy}_{x} \mathrm{Er}_{0.2-x} \mathrm{Bi}_{0.8} \mathrm{FeO}_{3}$ system, except for the values of the specific residual magnetizations of the composition $\mathrm{Er}_{0.2} \mathrm{Bi}_{0.8} \mathrm{FeO}_{3}$, which take values less than those in compositions with $0.05<x<0.15$ (Fig. 2). An increase in the absolute values of the coercive forces and residual specific magnetizations at all substitutions with respect to the initial matrix ( $\mathrm{BFO})$ is revealed. A change in the $\mathrm{R}-\mathrm{O}, \mathrm{Fe}-\mathrm{O}$ bond lengths and, as a result, the values of the $\mathrm{Fe}-\mathrm{O}-\mathrm{Fe}$ angles, leads to a change in the intensities of indirect exchange interionic interactions. With an increase in the content of $\mathrm{Er}^{3+}$ cations in the $\mathrm{Dy}_{x} \mathrm{Er}_{0.2-x} \mathrm{Bi}_{0.8} \mathrm{FeO}_{3}$ system, the average effective radius 

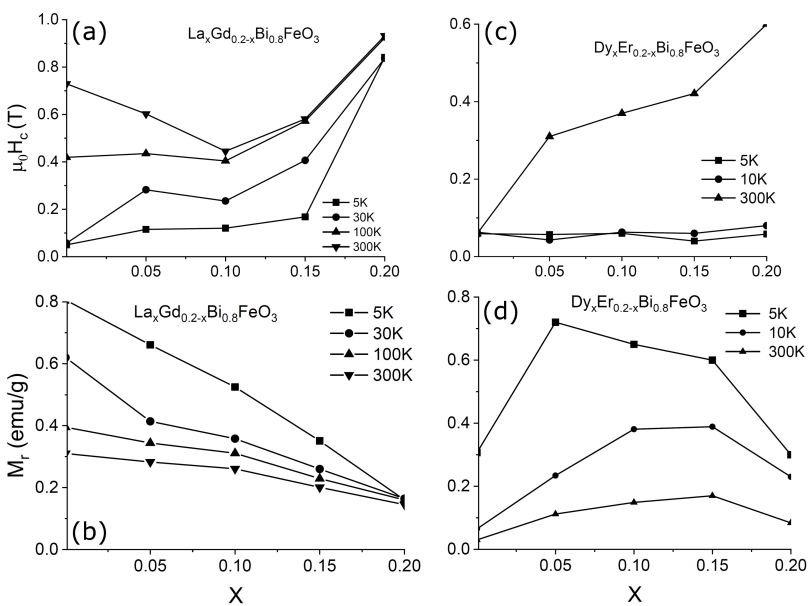

Fig. 1. Concentration dependences of coercive fields $\mu_{0} H_{c}$ and of specific residual magnetizations $M_{r}$ of LGBFO (a,b), and DEBFO (c,d) samples on the substitution degree at different temperatures.

of the rare-earth cation in the A sublattice decreases. This leads to a change in the distances between the ions of iron and oxygen and, as a consequence, to a change in the magnitudes of the "Fe-O-Fe" angles. To study the wFM-FM transition, respectively, the $M(T)$ dependence was calculated. Within the framework of the phenomenological model proposed by Hamad [15], the temperature dependence of magnetization $(M)$ in the vicinity of the Néel point $\left(T_{\mathrm{N}}\right)$ can be written as

$$
M=\frac{1}{2}\left(M_{i}-M_{f}\right) \tanh \left[A\left(T_{\mathrm{N}}-T\right)\right]+B T+C,
$$

where $M_{i}$ and $M_{f}$ are initial and final points of temperature range of wFM-AFM transition. The other coefficients are defined as

$$
\begin{aligned}
& A=2 \frac{B-S_{N}}{M_{i}-M_{f}}, \quad B=\left(\frac{\mathrm{d} M}{\mathrm{~d} T}\right)_{T_{i}}, \\
& S_{N}=\left(\frac{\mathrm{d} M}{\mathrm{~d} T}\right)_{T_{\mathrm{N}}}, \quad C=\frac{1}{2}\left(M_{i}+M_{f}\right)-B T_{\mathrm{N}} .
\end{aligned}
$$

To a first approximation, the value of the Néel point was determined by at least the first derivative $\mathrm{d} M / \mathrm{d} T$. Then its value was changed to obtain the best agreement between the model and experimental data. The "Fe-O-Fe" angles decrease with an increase in the replacement cations concentration. This leads to a decrease in the $T_{\mathrm{N}}$ values, the non-monotonic behavior of which in $x=0.05$ region is due to magnetization frustration caused by the coexistence of various phases. However, in general, there is an almost linear decrease in the Néel point with an increase in the degree of substitution (Fig. 3).

The concentration-related decrease in the Néel temperature values can be explained by the fact that, according to [16], the $T_{\mathrm{N}}$ value is directly proportional to the cosine of the valence bond angle. The "Fe-O-Fe" angles decrease with an increase in the replacement cations' concentration, which leads to a decrease in the $T_{\mathrm{N}}$ values, the

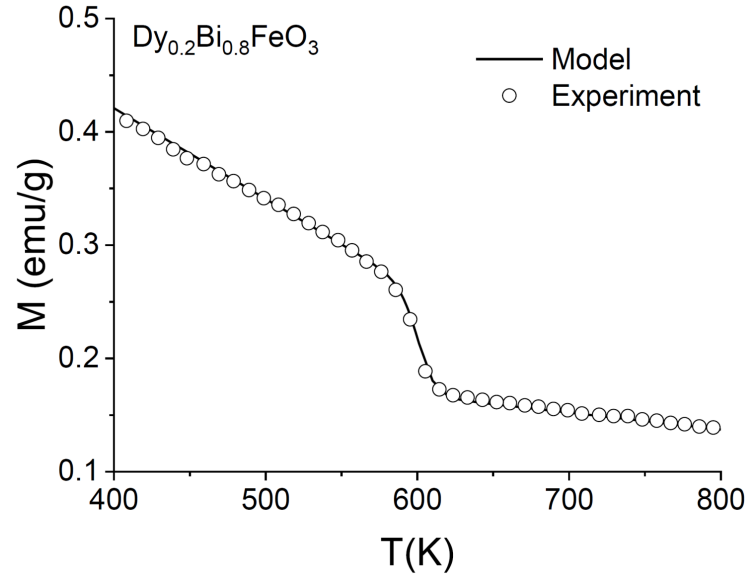

Fig. 2. Temperature dependences of specific magnetization $M(T)$ for the $\mathrm{Dy}_{0.2} \mathrm{Bi}_{0.8} \mathrm{FeO}_{3}$. The simulated curve obtained from (1) are indicated by a solid line.

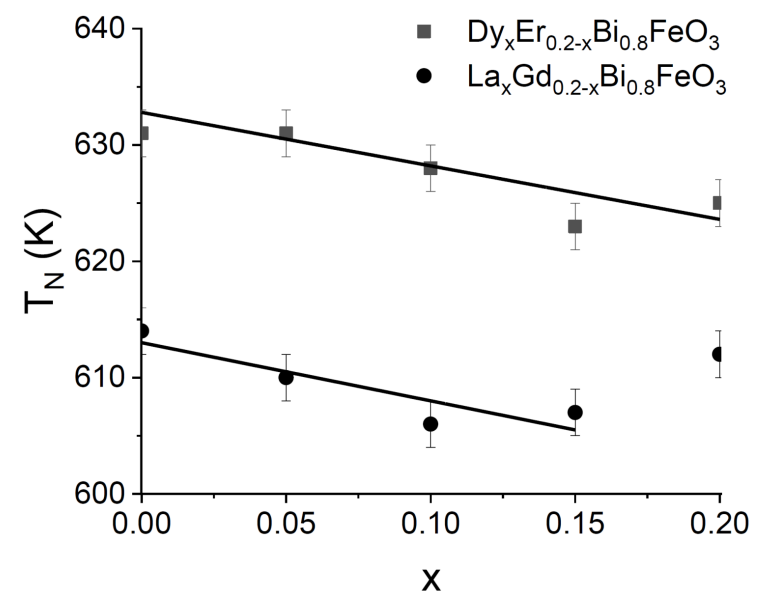

Fig. 3. Concentration dependences of Néel temperature for the $\mathrm{Dy}_{x} \mathrm{Er}_{0.2-x} \mathrm{Bi}_{0.8} \mathrm{FeO}_{3}$ and $\mathrm{La}_{x} \mathrm{Gd}_{0.2-x} \mathrm{Bi}_{0.8} \mathrm{FeO}_{3}$ samples.

non-monotonic behavior of which in the $x>0.10$ region is due to magnetization frustration caused by the coexistence of various phases, however, in general, there is an almost linear decrease in the Néel point with an increase in the degree of substitution.

\section{Conclusions}

X-ray diffraction and magnetic methods have been used for investigating the structure and magnetic properties of the ceramic $\mathrm{R}_{x} \mathrm{R}_{2.2-x} \mathrm{Bi}_{0.8} \mathrm{FeO}_{3}(\mathrm{R} 1, \mathrm{R} 2=\mathrm{La}$, $\mathrm{Gd}, \mathrm{Dy}, \mathrm{Er})$ samples with a concentration of $x=0-0.2$ prepared by a solid-state reaction method under cold pressing at high pressure $P=4 \mathrm{GPa}$. According to magnetic data, the temperature dependences of magnetization for the $\mathrm{R} 1_{x} \mathrm{R}_{0.2-x} \mathrm{Bi}_{0.8} \mathrm{FeO}_{3}$ multiferroics show magnetic "weak ferromagnetic-antiferromagnetic" 
at $T_{\mathrm{N}}=550-650 \mathrm{~K}$ phase transitions. The appearance of a weak ferromagnetism is caused by suppression of the spatial spin-modulated structure in course of an isovalent substitution of $\mathrm{Bi}^{3+}$ with $\mathrm{R}^{3+}$ cations in the $\mathrm{R} 1_{x} \mathrm{R} 2_{0.2-x} \mathrm{Bi}_{0.8} \mathrm{FeO}_{3}$. It has been established that the magnetic characteristics in the vicinity of the $T_{\mathrm{N}}$ point strongly depend on the concentration, temperature, and magnetic field. The results of the investigations of the magnetic properties for the $\mathrm{R} 1_{x} \mathrm{R} 2_{0.2-x} \mathrm{Bi}_{0.8} \mathrm{FeO}_{3}$ samples indicate the possibility of its practical application at temperatures above room temperature. The obtained data can be used to study the features of phase magnetic transitions in similar materials.

\section{References}

[1] G. Catalan, J.F. Scott, Adv. Mater. 21, 2463 (2009).

[2] D.C. Arnold, IEEE Trans. Ultrason. Ferroelectr. Freq. Contr. 62, 62 (2015).

[3] P. Suresh, P.D. Babu, S. Srinath, Ceram. Int. 42 4176 (2016).

[4] V.A. Khomchenko, L.C.J. Pereira, J.A. Paixão, J. Phys. D Appl. Phys. 44, 185406 (2011).

[5] X. Zhang, Y. Sui, X. Wang, J. Mao, R. Zhu, Y. Wang, Y. Liu, W. Liu, Z. Wang, J. Alloys Compd. 509, 5908 (2011).
[6] F.Z. Qian, J.S. Jiang, D.M. Jiang, W.G. Zhang, J.H. Liu, J. Phys. D Appl. Phys. 43, 025403 (2010).

[7] V.R. Palkar, Darshan C. Kundaliya, S.K. Malik, S. Bhattacharya, Phys. Rev. B 69, 212102 (2004).

[8] P. Priyadharsini, A. Pradeep, B. Sathyamoorthy, G. Chandrasekaran, J. Phys. Chem. Solids 75, 797 (2014).

[9] I.I. Makoed, A.A. Amirov, N.A. Liedienov et al., J. Magn. Magn. Mater. 489, 165379 (2019).

[10] V. Petříček, M. Dušek, L. Palatinu, Cryst. Mater. 229, 345 (2014).

[11] A.F. Ravinski, I.I. Makoed, K. Kokoshkevich, K.I. Yanushkevich, A.I. Galyas, V.V. Triguk, Inorg. Mater. 43, 860 (2007).

[12] G. Alvarez, J. Contreras, A. Conde-Gallardo, H. Montiel, R. Zamorano, J. Magn. Magn. Mater. 348, 17 (2013).

[13] R.D. Shannon, Acta Crystallogr. A 32, 751 (1976).

[14] A.K. Zvezdin, V.M. Matveev, A.A. Mukhin, A.I. Popov, Rare Earth Ions in Magnetically Ordered Crystals, Nauka, Moscow 1985 (in Russian).

[15] M.A. Hamad, J. Adv. Ceram. 1, 290 (2012).

[16] A. Bombik, B. Lesniewska, J. Mayer, A.W. Pacyna, J. Magn. Magn. Mater. 257, 206 (2003). 